\title{
Mis-Expression of a Cranial Neural Crest Cell-Specific Gene Program in Cardiac Neural Crest Cells Modulates HAND Factor Expression, Causing Cardiac Outflow Tract Phenotypes
}

\author{
Joshua W. Vincentz ${ }^{1, *}$, David E. Clouthier ${ }^{2}$ and Anthony B. Firulli ${ }^{1, *(D)}$ \\ 1 Herman B Wells Center for Pediatric Research, Departments of Pediatrics, Anatomy and Medical and \\ Molecular Genetics, Indiana Medical School, Indianapolis, IN 46202, USA \\ 2 Department of Craniofacial Biology, University of Colorado Anschutz Medical Campus, \\ Aurora, CO 80045, USA; david.clouthier@cuanschutz.edu \\ * Correspondence: jovincen@iu.edu (J.W.V.); tfirulli@iu.edu (A.B.F.)
}

Received: 30 March 2020; Accepted: 14 April 2020; Published: 20 April 2020

\begin{abstract}
Congenital heart defects (CHDs) occur with such a frequency that they constitute a significant cause of morbidity and mortality in both children and adults. A significant portion of CHDs can be attributed to aberrant development of the cardiac outflow tract (OFT), and of one of its cellular progenitors known as the cardiac neural crest cells (NCCs). The gene regulatory networks that identify cardiac NCCs as a distinct NCC population are not completely understood. Heart and neural crest derivatives (HAND) bHLH transcription factors play essential roles in NCC morphogenesis. The Hand1 ${ }^{\text {PA/OFT }}$ enhancer is dependent upon bone morphogenic protein (BMP) signaling in both cranial and cardiac NCCs. The Hand1 $1^{\text {PA/OFT }}$ enhancer is directly repressed by the endothelin-induced transcription factors DLX5 and DLX6 in cranial but not cardiac NCCs. This transcriptional distinction offers the unique opportunity to interrogate NCC specification, and to understand why, despite similarities, cranial NCC fate determination is so diverse. We generated a conditionally active transgene that can ectopically express DLX5 within the developing mouse embryo in a Cre-recombinase-dependent manner. Ectopic DLX5 expression represses cranial NCC Hand1 ${ }^{\text {PA/OFT }}$-lacZ reporter expression more effectively than cardiac NCC reporter expression. Ectopic DLX5 expression induces broad domains of NCC cell death within the cranial pharyngeal arches, but minimal cell death in cardiac NCC populations. This study shows that transcription control of NCC gene regulatory programs is influenced by their initial specification at the dorsal neural tube.
\end{abstract}

Keywords: HAND1; DLX5; BMPs; transcriptional regulation; neural crest; craniofacial defects; cardiac defects

\section{Introduction}

Congenital heart defects (CHDs) afflict roughly $1 \%$ of newborns and ultimately affect the quality of life of more than 1 million adults in the United States [1]. Many CHDs affect the cardiac outflow tract (OFT) [2]. A significant portion of CHDs can, therefore, be attributed to developmental dysfunction of one of the main developmental progenitors of the OFT, known as the neural crest cells (NCCs). NCCs migrate from the dorsal neural tube throughout the developing embryo [2]. Different NCC subpopulations differentiate into distinct tissue types. The cardiac NCCs differentiate into smooth muscle and connective tissue to form portions of the aorta, pulmonary artery, and nascent ventricular septum. Although NCC have been well studied, the gene regulatory networks that drive NCCs cells to 
delaminate from the neural tube, migrate to their destinations, and differentiate into their specified cell type identity are still not completely understood.

The bHLH transcription factors HAND1 and HAND2 are expressed in both cranial and cardiac NCCs and loss of function studies establish them as critical players in craniofacial and OFT development [3-6]. Hand1 NCC expression is observed post-NCC migration and resides within the ventral most cap of the pharyngeal arches that contribute to craniofacial structures as well as components of the OFT [7-9]. Hand2 expression is also observed post-NCC migration. However, Hand2 expression projects more dorsally and demarcates the entire ventral domain [6,8]. Hand1 NCC expression directly depends on HAND2 and BMP-signaling mediated transcriptional activity $[8,10,11]$. Hand2 NCC expression is dependent on the Endothelin-1 induced transcription factors DLX5 and DLX6, whose expression, with the exception of the Hand1 expression-marked ventral cap domain, overlaps with that of Hand2 [8]. HAND2 exhibits negative feedback upon Dlx5 and Dlx6, excluding $D l x 5 / 6$ expression from the ventral cap [6]. When Hand2 expression is deleted from NCCs, Dlx5/6 expression expands into the ventral cap, where it actively represses Hand1 expression [8]. Collectively, these findings are consistent with a complex regulatory mechanism that allows for sub specialization of NCC within the pharyngeal arches critical for craniofacial and OFT morphogenesis.

Single-cell NCC analyses have proposed a cardiac NCC differentiation cascade in which Dlx6 is transiently upregulated, followed by activation of $M s \times 2$ and Hand2, and then activation of downstream cardiac markers including Hand1 and Gata6 [12]. However, this gene expression profile describes both the cardiac NCCs and the distal cap cranial NCCs. In this study, we utilize a novel conditional gain-of-function Dlx5 allele to ectopically and persistently express DLX5 specifically within cranial NCCs. Using the NCC Cre driver, Wnt1-Cre, we demonstrate that, in cranial NCC populations, Hand1 expression is repressed, whereas cardiac NCC Hand1 expression is not directly affected. Significant amounts of cell death are observed in cranial NCC populations. However, cardiac NCCs exhibit less extensive apoptosis. This work advances our understanding of the unique transcriptional pathways at work within NCCs contributing to cranial and OFT tissues where gene expression within all NCC is interpreted differentially within specified NCC populations.

\section{Materials and Methods}

\subsection{Transgenic Mice}

The Indiana University Transgenic and Knock-Out Mouse Core generated the CAG-CAT-FLAGDlx5 transgenic mouse line (denoted henceforth as CAG-CAT-Dlx5) on a $\mathrm{C} 3 \mathrm{HeB} / \mathrm{FeJ}$ background. Genotyping for this allele is performed via PCR using the forward primer Dlx5(F) $5^{\prime}$-CGGGACGCTTTATTAGATGG-3' and the reverse primer Dlx5(R) 5'-TTGCATTGTTGGATTTCTGG-3', which produces a 465 bp control band and the reverse primer SV40pA(R) 5'-CCCCCTGAACCTGAAACATA-3', which produces a $295 \mathrm{bp}$ amplicon that detects the presence of the transgene. After a $5 \mathrm{~min}$ incubation at $95^{\circ} \mathrm{C}$, the PCR conditions run are $95{ }^{\circ} \mathrm{C} 30 \mathrm{~s}, 55^{\circ} \mathrm{C} 60 \mathrm{~s}$, and $72{ }^{\circ} \mathrm{C} 60 \mathrm{~s}$ for 36 cycles. Use and genotyping of Wnt1-Cre and $\mathrm{Gt}\left(\right.$ ROSA)26Sor ${ }^{\text {tm1Sor }}$ alleles are previously reported [13,14]. Embryos were not selected for sex and were evaluated blindly for all analyses. Mice and other reagents are available from the authors upon request.

\subsection{Cloning}

The generated Cre-activatable transgene CAG-CAT-Dlx5 was constructed by replacing the Myc-Twist1 cDNA of CAG-CAT-Twist construct [15] with the murine FLAGDlx5 cDNA.

\subsection{Bone and Cartilage Staining, X-Gal Staining and Histology}

Bone and cartilage staining was performed using Alizarin Red and Alcian Blue as previously described [6]. X-gal staining was performed as previously described [9,16-18]. 


\subsection{Lysotracker and TUNEL}

Cell death analysis on control and mutant embryos was performed as described [18,19]. Lysotracker (Life Technologies) was incubated with embryos as per the manufacturer's instructions. Embryos were imaged in a well slide on a Leica DM5000 B compound florescent microscope. TUNEL analyses were performed upon sectioned embryos using the ApopTag Plus Fluorescein in situ Apoptosis detection kit (S7111 Chemicon International) as per the manufacturer's instructions.

\subsection{Immunohistochemistry}

Immunohistochemistry was performed as previously described [17] using an antibody against TUBULIN $\beta 3$ ( $\beta$-TUBB3, Abcam). Images were collected on a Leica DM5000 B microscope and Leica Application Suite software.

\subsection{In Situ Hybridization}

Section in situ hybridizations were performed on 10- $\mu \mathrm{m}$ paraffin sections as described $[20,21]$. Antisense digoxygenin-labeled riboprobes were synthesized using T7, T3, or SP6 polymerases (Promega) and DIG-Labeling Mix (Roche) using the following plasmid templates: Dlx5, Dlx6, Hand2, Sox9 (provided by Benoit De Crombrugghe), and Ret (provided by Jean-Francois Brunet).

\subsection{Quantitative RT-PCR}

Total RNA was isolated from E11.5 mandibular pharyngeal arches using the High Pure RNA Isolation Kit (Roche). This RNA was then used to synthesize cDNA using the High-Capacity cDNA Reverse Transcription Kit (Applied Biosystems). For qRT-PCR, cDNA was amplified using TaqMan Probe-Based Gene Expression Assays (Applied Biosystems) and the QuantStudio 3 Real-Time PCR System (ThermoFisher). Normalization to Glyceraldehyde 3-phosphate dehydrogenase (GAPDH)was used to determine relative gene expression and statistical analysis automatically applied by the instrumental software. Significance of qRT-PCR results were determined by a two-tailed students t-test followed by post hoc Benjamini-Hochberg FDR correction as automatically calculated by the QuantStudio 3 qRT-PCR thermal cycler software analysis package.

\section{Results}

\subsection{NCC Expression of CAG-CAT-Dlx5 Results in Midface Clefting}

To test the hypothesis that mis-expression of a Dlx 5 within NCCs would result in craniofacial and cardiac NCC phenotypes, we generated a conditional Dlx5-gain-of-function mouse line (CAG-CAT-Dlx5, Figure 1A). We employed an NCC-specific Cre driver (Wnt1-Cre; [22]) to ectopically drive Dlx 5 expression within all NCCs (Wnt1-Cre;CAG-CAT-Dlx5 (Dlx5 NCC oe). In order to determine if persistent NCC expression of $D l x 5$ causes a NCC phenotype, we first looked at craniofacial structures at E16.5 (Figure 1). Compared with Cre-negative littermates (Figure 1B), the upper jaw (uj) of Dlx5 NCC oe embryos is underdeveloped and split along the midline, which results in a severe midfacial cleft that allows visualization of the tongue ( $t$, Figure 1C). The mandible (md) is also hypoplastic and misshaped (Figure 1D, E). This phenotype is similar to what is observed in HAND1 dimer mutant mice [9] but is far more severe than observed in embryos recently reported in which a Dlx 5 cDNA was inserted into the ROSA26 locus and then activated in NCCs $\left(N C C^{D l x 5}\right)$ [23]. 


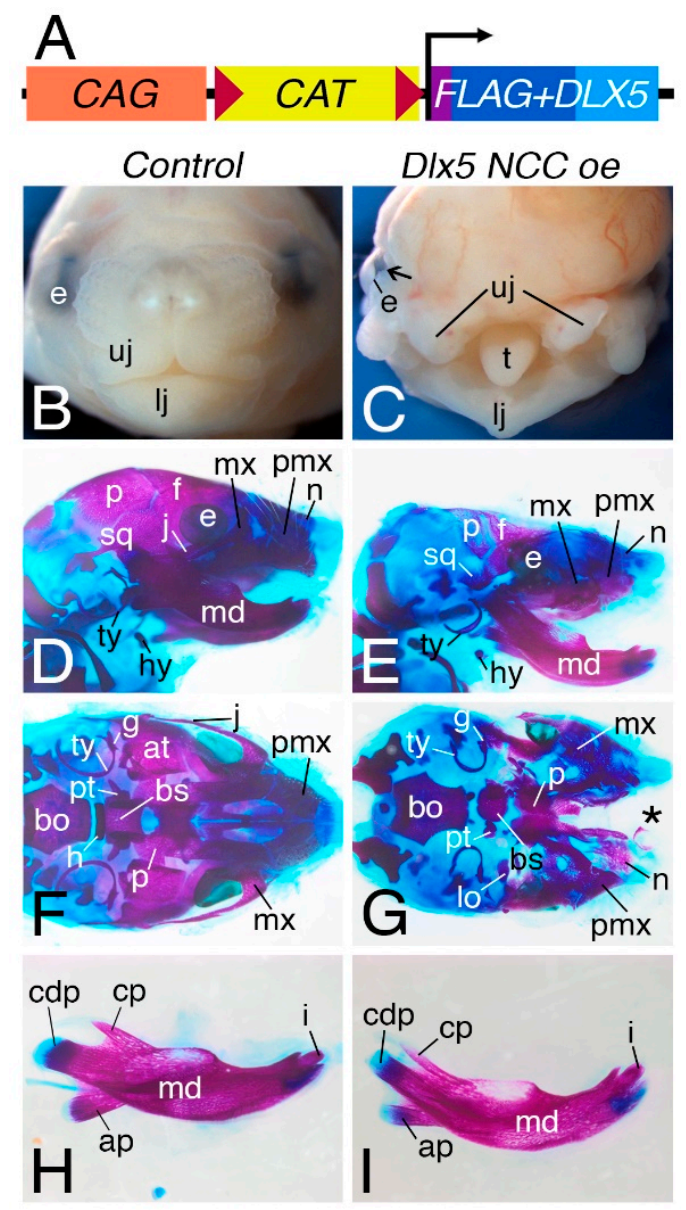

Figure 1. Neural crest cell (NCC)-specific Dlx5 overexpression results in midface clefting. (A) Diagram of the CAG-CAT-Dlx5 transgene. The CAG promoter drives expression of the Chloramphenicol acetyl transferase (CAT) gene that is flanked by loxP elements (triangles). Removal of Chloramphenicol Acetal transferase (CAT) via Cre-recombinase allows for persistent expression of $D l x 5$. (B,C). Anterior and ventral whole mount views, respectively, of E16.5 heads from Control (CAG-CAT-Dlx5) (B) and Wnt1-Cre; CAG-CAT-Dlx5 (Dlx5 NCC oe) (C) embryos. Compared to control embryos, the midface of Dlx5 NCC oe embryos contain a large cleft separating the upper jaw (uj) into left and right sides and exposing the tongue (t). The lower jaw (lj) also appears misshapen and a coloboma (arrow) is present. (D-I). E18.5 control (B,D,F,H) and Dlx 5 NCC oe (C,E,G,I) embryos stained with Alizarin Red and Alcian Blue to visualize bone and cartilage, respectively. $(\mathbf{D}, \mathbf{E})$ Lateral views of skulls. In control embryos, the parietal (p) and frontal (f) are observed, which abut the squamosal (sq) bone (D). In the jaw region, the nasal (n), premaxilla (pmx), and maxilla $(\mathrm{m})$ bones are observed, with the zygomatic process of the maxilla abutting the jugal ( $\mathrm{j}$ ) bone of the zygomatic arch. The tympanic ring (ty) bone is also observed. In Dlx 5 NCC oe mice, the parietal, frontal bones are hypoplastic, as is the tympanic ring and squamosal bones (E). The premaxilla, maxilla, and jugal bones are dysmorphic, though the mandible appears relatively normal. (F,G) Ventral view of skulls with the mandible removed. In contrast to control embryos (F), most skull base bones in Dlx5 NCC oe embryos are hypoplastic, including the basisphenoid (bs), pterygoids (pt), and alisphenoids (al). The palatine (p) bones fuse along the midline but are also hypoplastic, as are the tympanic rings $(\mathbf{G})$. The dysmorphology of the maxilla along the midline is also apparent, along with the large midline cleft that now exists $\left({ }^{*}\right)$. $(\mathbf{H}, \mathbf{I})$ Lateral (intra-oral) view of the left mandible (md). The coronoid (cp), condylar (cdp), and angular (ap) processes of the mandible are present in both control (H) and Dlx5 NCC oe (I) embryos, as are the incisors (i). bo, basisoccipital bone; e, eye; hy, hyoid bone. 
To determine how these changes were reflected in near term embryos, E18.5 Dlx5 NCC oe and Control (Cre-negative CAG-CAT-Dlx 5 littermates) embryos were stained with Alizarin Red and Alcian Blue to visualize bone and cartilage, respectively. Compared to control embryos (Figure 1C,E), the NCC-derived bones of the skull vault (frontal, $\mathrm{f}$ ) and nasal, $\mathrm{n}$ ) and skull base bone (basisphenoid (bs), alisphenoid (al), and palatine (p) bones) are hypoplastic or absent in Dlx5 NCC oe embryos (Figure 1D,F). In addition, there is a large midfacial cleft in which the two halves of the premaxilla (pmx) fail to meet at the midline. This cleft extends back through the palatal processes of the maxilla $(\mathrm{mx})$ but does not affect fusion of the palatine bones. The maxilla bones are quite dysmorphic, which makes it impossible to determine if they represent a homeotic transformation into more a mandible-like shape as observed in the $N C C^{D l x 5}$ mice [23]. However, these changes are similar to those observed when an Endothelin-1 (Edn1) cDNA is driven in NCCs using Wnt1-Cre mice [24,25]. In one Chicken b-actin (CBA)-Edn1;Wnt1-Cre line, embryos have a classic homeotic transformation of the maxilla into a mandible-like structure while embryos from another line have a large midfacial cleft. Since there is not a significant difference in mature EDN1 by Enzyme-Linked ImmunoSorbent Assay (ELISA) between the two lines, the differences likely reflect insertion site dynamics, with the varying phenotypic outcome reflecting a range of EDN1 action in NCCs. Overall, the phenotype of Dlx 5 NCC oe embryos indicates that DLX5 has several unrecognized functions during early NCC patterning. A more detailed analysis of this is being reported elsewhere.

\subsection{NCC Expression of CAG-CAT-Dlx5 Down Regulates Hand1 Ventral Cap Expression}

DLX5 regulates gene expression in post-migration cranial NCCs and, although expressed in cardiac NCCs, is not required for OFT morphogenesis, as heart defects are not reported in Dlx5;Dlx6 double knockout mice [12,23-27]. DLX5 negatively regulates Hand1 expression [8] and positively regulates Hand2 expression [6,28] in cranial NCCs. To ensure that the Cre-activated transgene is functional, we first intercrossed CAG-CAT-Dlx 5 mice with the Hand2-Cre driver (Figure 2) [3]. E10.5 whole mount in situ hybridization of $D l x 5$ shows normal robust dorsal expression where the yellow line demarks its ventral most expression and the white line shows the ventral most cap of the arch of control embryos (Figure 2A). Cre-activation of the CAG-CAT-Dlx5 allele reveals a noticeable expansion of Dlx5 expression ventrally such that the space between the yellow and white lined marked boundaries is noticeably reduced, which indicates the efficacy of the Cre-inducible CAG-CAT-Dlx5 allele (Figure 2B). We next intercrossed CAG-CAT-Dlx 5 mice with our Hand1 ${ }^{\text {PA/OFT }}$-lacZ reporter line, which we have previously shown is sensitive to DLX5 negative regulation [8]. At E11.5, control embryos show expected cranial and cardiac NCC expression as well as the second heart field derived myocardium of the myocardial cuff (Figure 2C). Most notably, the ventral most NCC of the mandibular arch strongly expresses the Hand1 $1^{\text {PA/OFT }}$-lacZ reporter $(1$, arrowhead, Figure $2 \mathrm{C}, \mathrm{n}=4)$. When Hand1 $1^{\text {PA/OFT }}$-lacZ reporter expression is combined with the Wnt1-Cre; CAG-CAT-Dlx5 alleles, significantly reduced $\beta$-galactosidase staining is observed within the ventral cap domain of arch 1 (Figure 2D arrowhead, $\mathrm{n}=4$ ). Transverse sections through the cardiac OFT reveal robust $\beta$-galactosidase staining within the NCC mesenchyme and OFT myocardium (Figure 2E arrow). In Dlx 5 NCC oe; Hand1 ${ }^{\text {PA/OFT }}$-lacZ mice, the $\beta$-galactosidase staining intensity is unchanged. However, the ventral boundary of the $\beta$-galactosidase positive NCCs is more dorsal (Figure 2F arrow). 


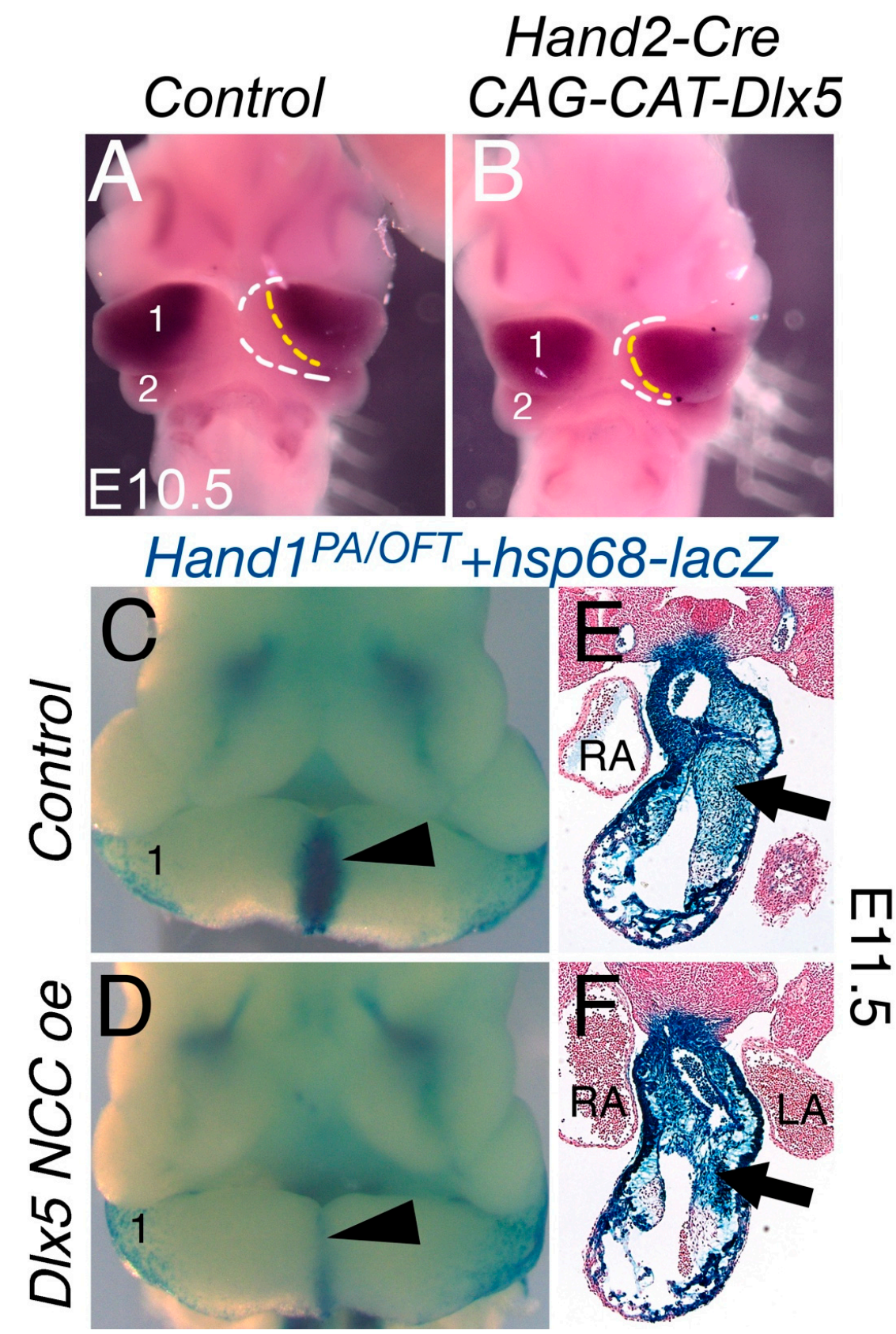

Figure 2. NCC-specific $D l x 5$ overexpression represses Hand1 ${ }^{\text {PA/OFT }}$ expression. (A) E10.5 wholemount in situ hybridization of Dlx 5 in Control and Hand2-Cre; CAG-CAT-Dlx5 embryos. The yellow dotted line marks the ventral most domain of expression and the white dotted line marks the ventral most aspect of the first mandibular arch (1). (B) Hand2-Cre-activated Dlx5 transgene expression reveal a measurable ventral extension of Dlx5 expression into the ventral arch (reduced space between yellow and white lines). (C) Wholemount $\beta$-galactosidase staining from the Hand1 ${ }^{\text {PA/OFT }}$ transgene. The arrowhead marks distal cap expression. (D) Hand1 $1^{\text {PA/OFT }}$-lacZ transgene expression on Dlx 5 NCC oe (Wnt1-Cre; CAG-CAT-Dlx5) alleles. Visibly reduced $\beta$-galactosidase staining within the distal arch domain is observed. (E) Transverse section through the cardiac OFT of Hand1 ${ }^{\text {PA/OFT }}$ mice stained for $\beta$-galactosidase. Staining is robustly observed within cardiac NCC (arrow) and myocardial cuff. (F) Hand1 $1^{\text {PA/OFT }}$-LacZ transgene expression on Dlx5 NCC oe alleles. Staining is similar in intensity to control embryos. However, the ventral extension of $\beta$-galactosidase positive NCC is moved dorsally (arrow). 


\subsection{Dlx5 NCC oe OFTs Present Persistent Truncus Arteriosus (PTA)}

Disruption of Hand factor expression causes cardiac defects within the OFT [4,28]. To determine whether persistent $D l x 5$ expression within cardiac NCCs alters OFT morphogenesis and to assay Hand1 OFT expression, we looked at E16.5 hearts (Figure 3). In control (CAG-CAT-Dlx5; Hand1 ${ }^{\text {PA/OFT }}$-lacZ) E16.5 hearts, OFT formation appears normal, wherein the pulmonary artery (PT) directly connects with the right ventricle (RV) and the aorta (Ao) directly connects with the left ventricle (LV, Figure 3A-C). Hand1 $1^{\text {PA/OFT }}$-lacZ expression, visualized by $\beta$-galactosidase staining, is detectable within the smooth muscle wall of the aorta and within myocardial cuff cardiomyocytes. In contrast, Dlx 5 NCC oe mutants present with either a single OFT vessel, which is a condition termed persistent truncus arteriosus (PTA, Figure 3D and $E, n=6 / 10$ ), or with a double outlet right ventricle (DORV), wherein the Ao connects directly with both right ventricle (RV) and left ventricle (LV) (Figure 3F-H, n =3/10). Hand1 $1^{P A / O F T}$-lacZ expression is observed within the aortic smooth muscle and cuff myocardium. Summary of the encountered phenotypes is presented in Table 1.

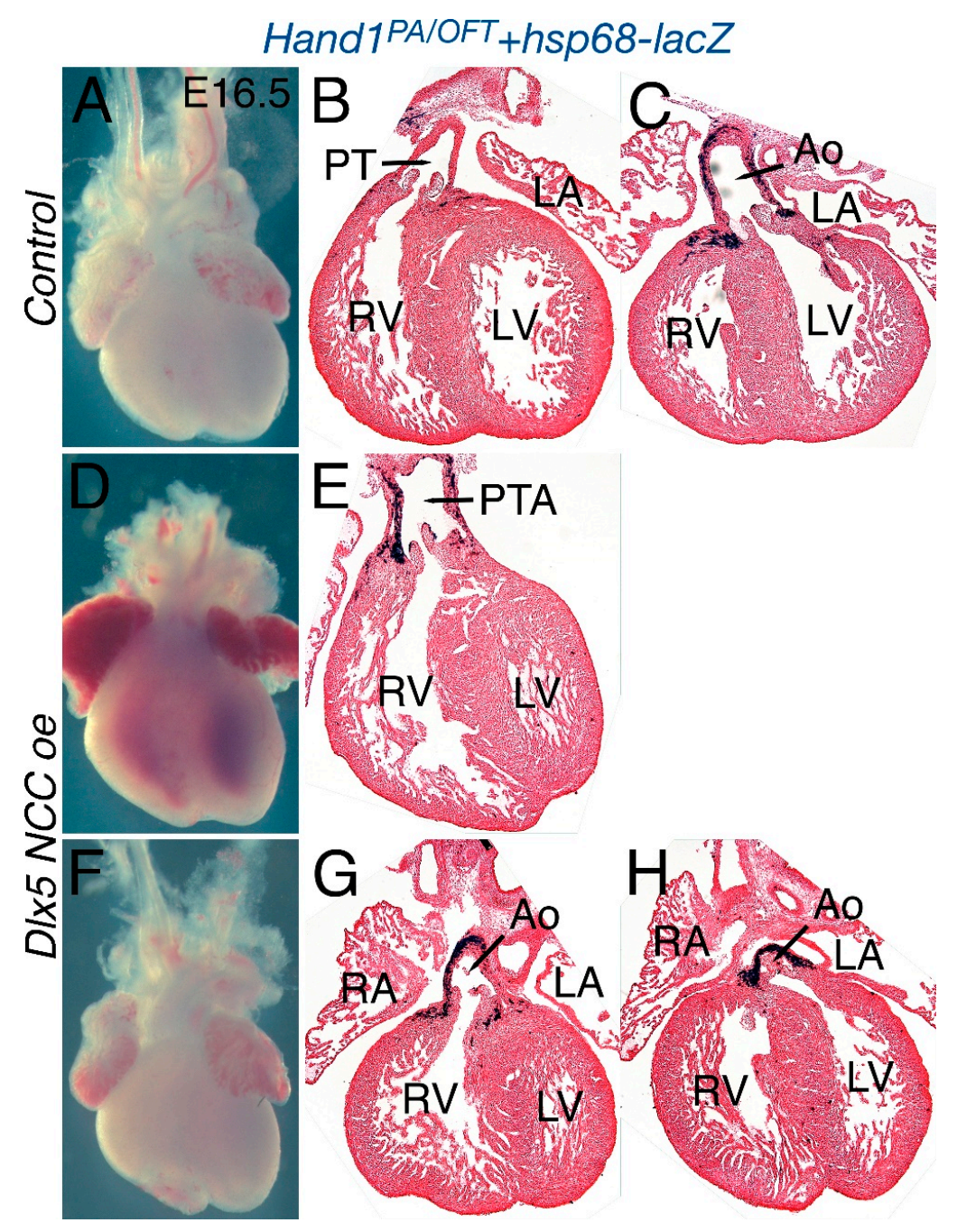

Figure 3. Phenotypic analysis of Dlx5 NCC oe hearts at E16.5. Whole mount (A) and frontal sections (B,C) of control (CAG-CAT-Dlx5;Hand1 ${ }^{\text {PA/OFT }}$-lacZ) heart showing patent septation and proper alignment of the pulmonary trunk (PT) and aorta (Ao) with the right (RV) and left (LV) ventricles. Wholemount Dlx5 NCC oe hearts at E16.5 reveal persistent truncus arteriosus (PTA, D,E) or double outlet right ventricle (F-H) wherein the aorta is connected to both RV: right ventricle and LV; left ventricle, RA: right atria, LA: left atria. 
Table 1. Outflow tract (OFT) defects observed in Dlx5 neural crest cell (NCC) oe mutants.

\begin{tabular}{ccccc}
\hline Genotype & n & PTA + VSD & DORV + VSD & Phenotypically Normal \\
\hline Wht1-Cre & 1 & $0(0 \%)$ & $0(0 \%)$ & $1(100 \%)$ \\
CAG-CAT-Dlx5 & 3 & $0(0 \%)$ & $0(0 \%)$ & $3(100 \%)$ \\
$\begin{array}{c}\text { CAG-CAT-Dlx5; } \\
\text { Wnt1-Cre }\end{array}$ & 10 & $6(60 \%)$ & $3(30 \%)$ & $1(10 \%)$ \\
\hline
\end{tabular}

DORV, double outlet right ventricle, PTA, persistent truncus arteriosus, and VSD, ventricular septal defect.

\subsection{NCC Migration into the OFT Is Limited in Dlx5 NCC oe Mice}

The PTA and DORV observed in Dlx5 NCC oe mutants could be due to do altered NCC morphogenesis, increased NCC cell death, or lack of normal NCC migration. To determine what mechanisms are involved, we first performed a Wnt1-Cre lineage analysis between E9.5 and E11.5 to look for migration of Wnt1-Cre lineage cells within the OFT (Figure 4). In both E9.5 (28 and 29 somite) and E10.5 (36 somite) embryos, both the first and second pharyngeal arches are robustly positive for Wnt1-Cre marked NCCs (Figure 4A,C, arrows). Cardiac NCCs are also robustly present dorsal to the OFT (bracket) and can be seen entering the OFT (arrowhead, Figure 4A,C). In Dlx5 NCC oe mutants, Wnt1-Cre marked NCCs are also observed. However, $\beta$-galactosidase staining is less robust (Figure 4B,D). First and second phalangeal arches are smaller (Figure 4B,D, arrows). The population of cardiac NCCs dorsal to the OFT is smaller (Figure 4B,D, bracket) and the Wnt1-Cre marked NCCs visible within the OFT are clearly diminished (Figure $4 \mathrm{~B}, \mathrm{D}$, arrowheads).

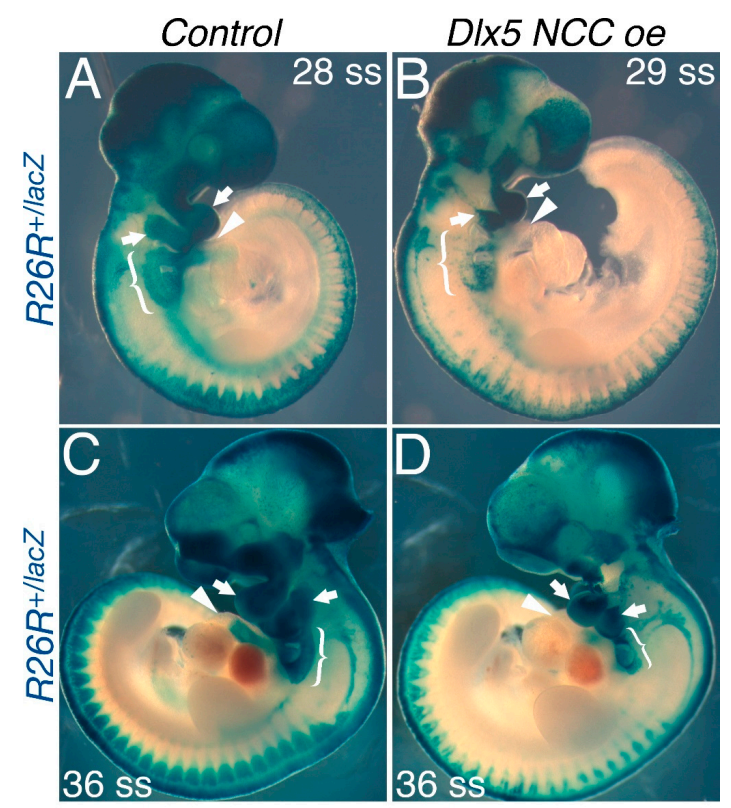

Figure 4. NCC lineage analysis of Dlx5 NCC oe E9.5 and E10.5 embryos. (A) E9.5 Wnt1-Cre; R26R lacz/+ embryo stained for $\beta$-galactosidase activity. Blue stained cells represent NCC migration through the embryo. Cranial NCCs populating the first and second pharyngeal arches are noted by arrows. Cardiac NCCs occupying the caudal pharyngeal arches are denoted by brackets. NCCs within the outflow tract (OFT) are denoted by arrowheads. (B) NCC lineage analysis in Dlx5 NCC oe embryos shows reduced first and second pharyngeal arch size (arrows) and reduced cardiac NCC number dorsal to and within the OFT. (C) Control and (D) Dlx 5 NCC oe embryo NCC lineage analysis at E10.5 demonstrate that the cranial arches are hypoplastic in Dlx 5 NCC oe embryos when compared to control embryos (arrows). Reduced numbers of cardiac NCCs (brackets) and NCCs within the OFT (arrowheads) are also observed. 
3.5. Dlx5 NCC oe Embryos Exhibit NCC Cell Death Within the Neural Tube, Pharyngeal Arches but Minimally Within the OFT

To determine whether Wnt1-Cre marked NCC reduction within the pharyngeal arches reflects solely migration defects and not NCC cell death, we performed lysotracker staining to assess cell death in E9.5 and E10.5 Dlx 5 NCC oe embryos. In E9.5 controls, developmentally normal cell death is observed with regions of the head as well as tissues located dorsally to the OFT (Figure 5A, bracket). Cell death within the pharyngeal arches is minimal (arrow Figure $5 A, n=4$ ). In contrast, significant cell death is observed at the dorsal neural tube (arrowheads) and within the first and second pharyngeal arches (arrow robust positive staining within the arches, Figure $5 B, n=4$ ). Cell death in the caudal pharyngeal arches was not appreciably affected in E9.5 Dlx5 NCC oe embryos (Figure 5A,B, brackets, $\mathrm{n}=4)$. No significant cell death is observed within the heart. At E10.5, control (CAG-CAT-Dlx5) embryos displayed domains of lysotracker-positive cells within the proximal rostral pharyngeal arches (Figure 5C, white arrows). Although the pharyngeal arches are now hypoplastic at this stage, the lysotracker-positive cells of proximal rostral pharyngeal arches of Dlx 5 NCC oe littermates are not observed (Figure 5D, white arrows). Continued cell death along the dorsal neural tube is still evident (arrowheads, Figure 5D). To characterize this aberrant cell death in closer detail, we performed TUNEL analyses upon embryonic sections at E10.5. (Figure 5E-H). Compared to control embryos, extensive cell death in the dorsal neural tube is visible in the Dlx5 NCC oe embryos at E10.5 (compare Figure 5E,F, arrowheads, $\mathrm{n}=5$ ). The cranial pharyngeal arches are also clearly hypoplastic in E10.5 Dlx5 NCC oe embryos. No significant changes in cell death are observed in E10.5 OFT of Dlx5 NCC oe embryos when compared to control embryos. Together, this data shows that decreased NCC contribution to the pharyngeal arches and OFTs of Dlx 5 NCC oe embryos is mechanistically likely the result of the increased NCC death observed during NCC migration that initiates at their point of origin within the dorsal neural tube.

To determine whether gene expression is altered in the Dlx 5 NCC oe NCCs that contribute to the OFT, we looked at the expression of the cardiac NCC and OFT myocardium marker Hand2 [28,29] and the NCC marker Sox9 [30,31] (Figure 6). Expression of Hand2 is modest within the ventricular myocardium of the RV and LV and more robust within the endocardium, the myocardial cuff myocardium, and cardiac NCC (arrow) of control E10.5 hearts (Figure 6A). In Dlx 5 NCC oe hearts, expression within the endocardium, ventricles, and myocardial cuff is similar to that of control hearts. However, expression within the cardiac NCC is diminished (arrow, Figure 6B). Robust Sox9 expression is observed within control OFT NCCs (arrow, Figure 6C), whereas Sox9 expression with Dlx5 NCC oe OFT NCCs is significantly reduced, which results from decreased expression and / or less Sox9-expressing NCCs (arrow, Figure 6D).

\subsection{Persistent Dlx5 Expression Does Not Induce NCCs to Adopt a Neuronal Cell Fate}

Loss of TWIST1 function induces NCCs to differentiate along a neuronal cell fate [13]. Within the OFT, Twist1-null cardiac NCCs organize into ganglia-like structures and express a number of neuronal genes [13]. Additionally, these trans-differentiating cardiac NCCs express Hand1 [13]. Crossing the Hand1 ${ }^{\text {PA/OFT }}$-lacZ reporter onto a Twist1 ${ }^{-f x}$, Wnt1-Cre(+) background (Twist1 NCC CKO) reveals that these NCCs are specifically marked by Hand1 $1^{\text {PA/OFT }}$ enhancer activity (Figure 7B, arrowheads). Intriguingly, these ganglia-like structures also express Dlx 5 (Figure 7D, arrowhead, $\mathrm{n}=3$ ), which is not detectable in the cardiac NCCs of control OFTs (Figure 7C). To determine whether persistent Dlx 5 expression within the NCCs promotes cardiac NCC populations to differentiate along a neuronal path, we looked at the expression of the pan-neuronal marker Class III $\beta$-TUBULIN (TUBB3) [32] and the receptor tyrosine kinase Ret, which marks NCC-derived neurons [33]. TUBB3 immunostaining of E11.5 embryos on the Hand1 ${ }^{\text {PA/OFT }}$-lacZ reporter background revealed no detectable TUBB3 protein within OFT NCCs of either control or Dlx5 NCC oe embryos (Figure 7E,F, $\mathrm{n}=2$ ). Ret in situ hybridization of E10.5 embryos yielded similar findings (Figure $7 \mathrm{G}, \mathrm{H}, \mathrm{n}=4$ ). These results show that, although $D l \times 5$ 
expression is induced in ectopic neurons within Twist1-null cardiac NCCs, ectopic Dlx 5 expression within the cardiac NCCs is not sufficient to induce neurogenesis.

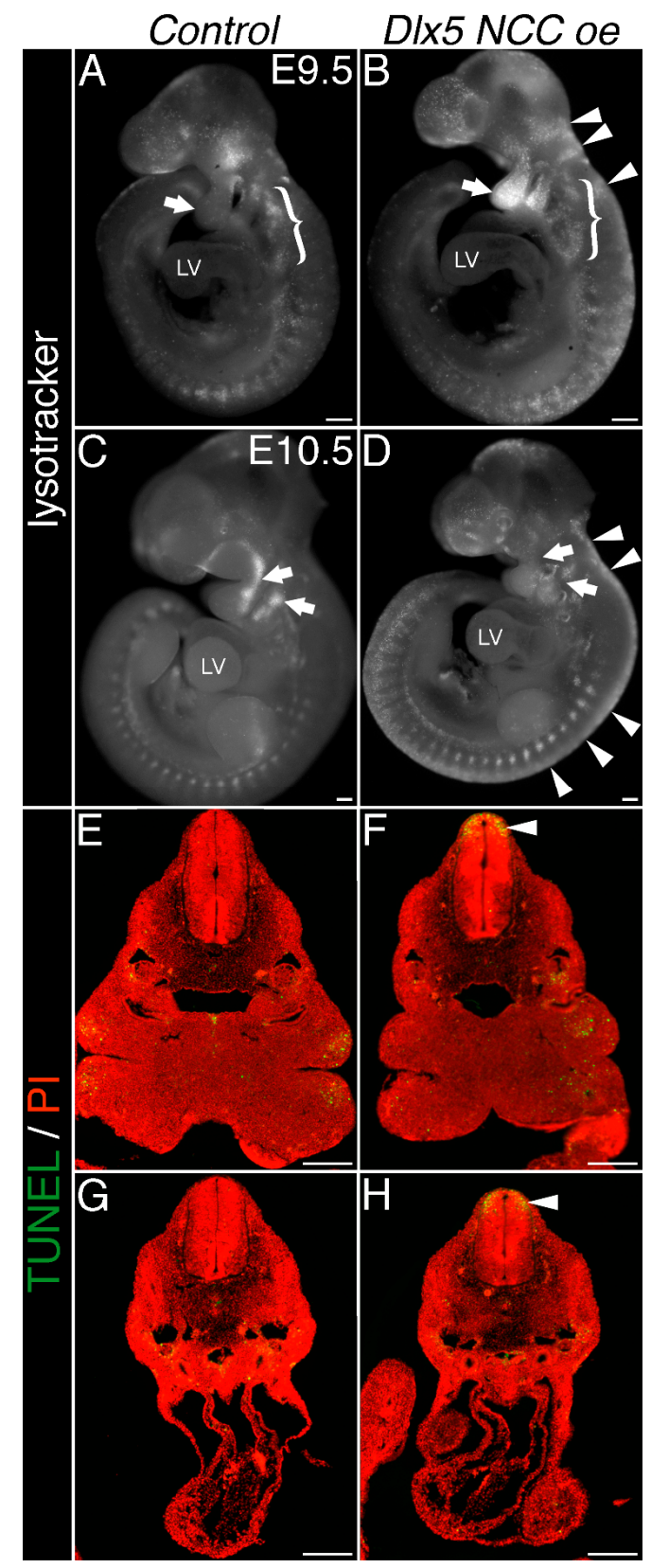

Figure 5. Lysotracker and Terminal deoxynucleotidyl transferase. dUTP nick end labeling (TUNEL) staining of Dlx5 NCC oe embryos. (A) E.9.5 control embryo showing normal cell death within the head and tissues dorsal to the OFT (brackets). No significant apoptosis is observed within the first and second pharyngeal arches (arrow). (B) E9.5 Dlx 5 NCC oe embryo showing visible cell death within the neural tube (arrowheads) and, most notably, within the first and second pharyngeal arches (arrow). (C) E10.5 control embryo showing normal cell death. Arrows denote cell death within the proximal rostral pharyngeal domain of the first and second pharyngeal arches. (D) E10.5 Dlx 5 NCC oe embryo showing persistent dorsal neural tube cell death, whereas first and second pharyngeal arches show less apoptosis than controls. (E) and (F) E10.5 TUNEL stained control and Dlx5 NCC oe embryos. Elevated cell death within the neural tube of Dlx5 NCC oe embryos is clearly visible (arrowhead), as are the hypoplastic first and second pharyngeal arches. (G,H) more caudal sections showing minimal cell death within the cardiac OFT in control and Dlx5 NCC oe embryos. Scale bars $=200 \mu \mathrm{m}$. 


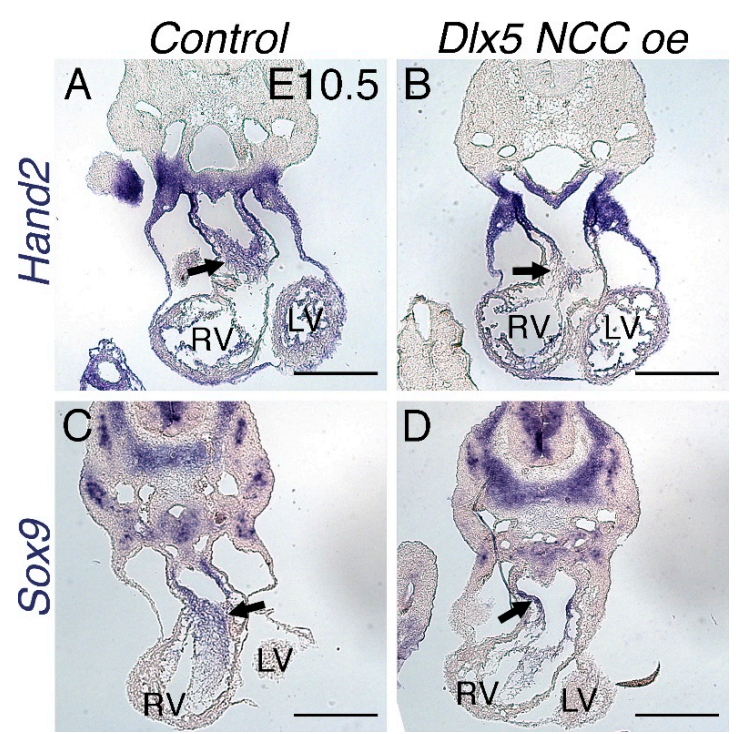

Figure 6. Expression of the OFT markers Hand2 and Sox 9 is reduced within Dlx5 NCC oe embryos. (A,B) In situ hybridization showing expression of Hand2 within control (A) and Dlx5 NCC oe (B) E10.5 embryo hearts. (C,D) E10.5 control (C) and Dlx5 NCC oe (D) embryos showing Sox 9 mRNA expression. Arrows denote cardiac NCC. Scale bars $=250 \mu \mathrm{m}$.

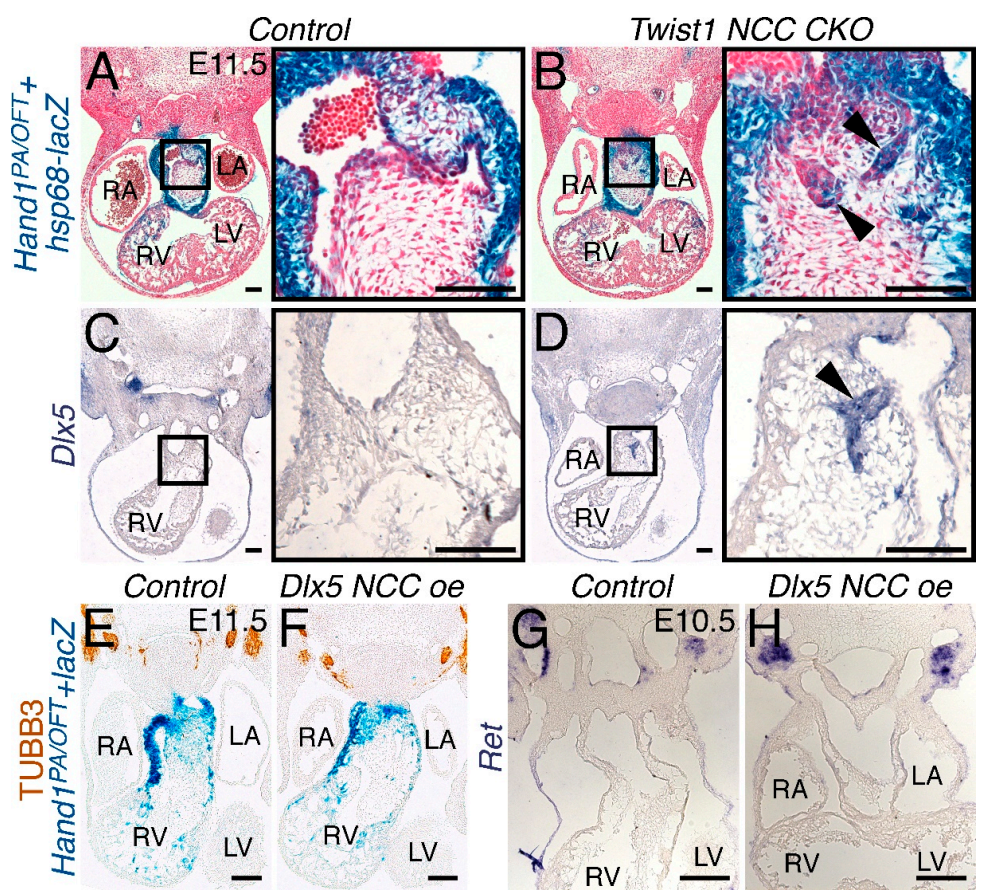

Figure 7. Ectopic Dlx5 expression within the NCC is not sufficient to induce neurogenesis. (A,B) E11.5 Hand1 ${ }^{\text {PA/OFT }}$-lacZ stained control (A) and Wnt1-Cre(+); Twist1-/fx mutant (B) embryos showing transgene-positive ectopic ganglia within the cardiac NCCs occupying the OFT. (C,D) In situ hybridization to detect Dlx5 mRNA shows no expression in control OFTs (C) but does show robust expression within the trans-differentiated cardiac NCCs of Twist1 conditional knockouts (D). Immunohistochemistry of pan-neuronal TUBB3 in both control (E) and Dlx5 NCC oe (F) sections reveals no ectopic neuronal staining in the E11.5 OFT. In situ hybridization to detect Ret mRNA similarly reveals no neuronal cells within the OFTs of E10.5 control (G) or Dlx 5 NCC oe $(\mathbf{H})$ embryos. Scale bars $=100 \mu \mathrm{m}$. 


\subsection{Persistent Dlx5 Expression within NCCs Downregulates Dlx6 Expression}

Signaling from the Endothelin receptor type A (EDNRA) induces $D l x 5$ expression in the pharyngeal arches [34]. In order to see whether DLX5 overexpression had regulatory effects upon other Endothelin 1-induced genes, we looked at expression of Hand2 and the related homeobox transcription factor Dlx6 in the pharyngeal arches of the E10.5 control and Dlx 5 NCC oe embryos (Figure 8). In situ hybridization of Hand 2 mRNA revealed that Hand2 expression appears unchanged within the first pharyngeal arch (1) in Dlx 5 NCC oe embryos (Figure $8 \mathrm{~A}, \mathrm{~B}, \mathrm{n}=3$ ). This is confirmed quantitatively by qRT-PCR (Figure $8 \mathrm{E}$, $\mathrm{n}=6$ ). In contrast, $D l x 6$ expression is robust within the first pharyngeal arch of control embryos but is significantly reduced in in Dlx5 NCC oe embryos (Figure 8C,D,E). Hand1, which is known to be negatively regulated by DLX5 [8], is also significantly downregulated (Figure 8E).
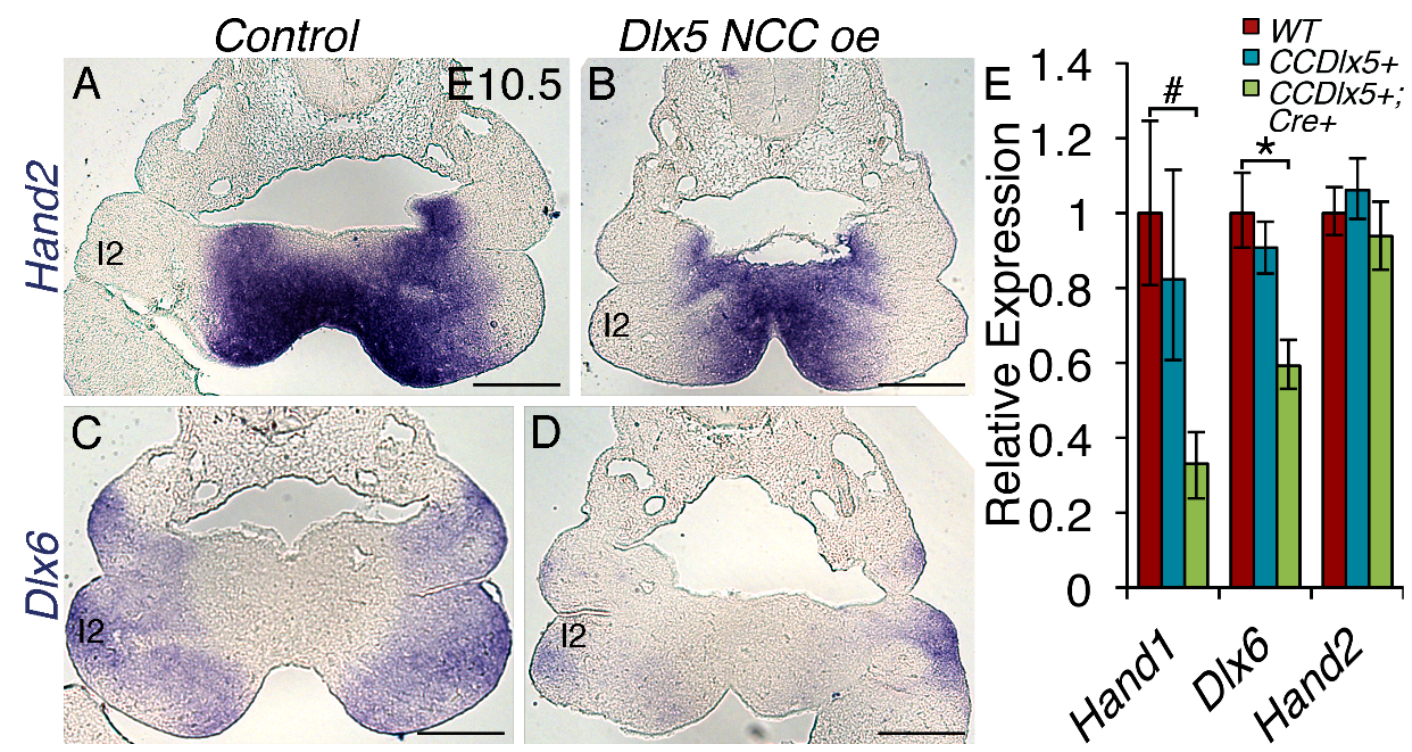

Figure 8. Dlx 5 NCC overexpression downregulates Dlx6 within the first pharyngeal arch. (A, B) Hand2 in situ hybridization showing normal first arch expression in both control (A) and Dlx5 NCC oe E10.5 embryos. Dlx6 in situ hybridization showing altered first arch expression when comparing control (C) and Dlx5 NCC oe embryos (D). (E) qRT-PCR analyses of Hand1, Dlx6, and Hand2 expression from E10.5 first pharyngeal arches. Significant decreases in Hand1 (\# $p \leq 0.01)$ and Dlx6 $\left({ }^{*} p \leq 0.05\right)$ are observed. No significant changes in the Hand2 message are detected. Scale bars $=250 \mu \mathrm{m}$.

\section{Discussion}

NCCs are a dynamic and multipotent cell population that, during embryogenesis, migrate ventrally from the neural tube to contribute to organ morphogenesis [35]. Major components of the cardiac OFT and nearly the entire vertebrate facial complex are NCC-derived. Dysregulation of these NCC populations result in the majority of congenital abnormalities encountered in humans. In this study, we set out to interrogate how NCC gene regulatory networks that include HAND transcription factors facilitate NCC specialization into specific tissue fates. Hand1 and Hand2 mark both cranial and cardiac NCC populations [36,37], exhibit genetic interactions that, when disrupted, result in a phenotype $[4,19,21,38]$, and set up tissue boundaries that are essential normal tissue morphogenesis within the post migration NCCs occupying the pharyngeal arches $[6,8]$.

The cranial transcriptional enhancers that drive Hand1 and Hand2 within NCC as well as the cardiac NCC transcriptional enhancer for Hand1 are established [2,5,39]. Analysis of these enhancers has revealed transcriptional inputs from both Endothelin Receptor A EDNRA (through DLX5 and DLX6) and BMP signaling (through SMADs 1/5/8) as well as direct and required regulation of Hand1 by HAND2 and DLX5/6 [8,10,39]. Given the spatial changes in Hand1 expression that result from 
BMP gain-of-function and HAND2 loss-of-function, ectopic expression of DLX5 within all NCC was performed to look at the effects on cranio-facial and OFT formation.

The first observation that is noted is that Dlx 5 NCC oe mice exhibit severe midface clefting (Figure 1 ). We speculate that mechanistically this is likely the result of increased cranial NCC apoptosis (Figure 4). Hand1 phospho mutant conditional knock-in mice exhibit a similar craniofacial phenotype [9]. Although Hand1 NCC loss-of-function mice exhibit no observable phenotypes [4], it is clear that Hand1 is down regulated within Dlx 5 NCC oe mice (Figures 2 and 8). If other potential HAND1 bHLH partners are also transcriptionally regulated, the combination of these changes to the bHLH gene regulatory networks via alteration of the bHLH dimer pool available to form transcriptional dimer complexes could account for the similar phenotypes. Of note, a similar study employing a Rosa locus Dlx 5 knockin was recently reported [12,23-26] and showed short snout, open eyelids, misaligned vibrissae, and a cleft palate with no clear signs of palatine rugae. In our study, using a traditional transgenic insertion increases severity and is likely the result of increased Dlx 5 expression in our model.

The Hand1 ${ }^{\text {PA/OFT }}$ enhancer drives expression in both cranial and cardiac NCCs, and DLX5 and DLX6 directly repress Hand1 ${ }^{\text {PA/OFT }}$ enhancer activity [8]. However, Dlx5 and Dlx6 are not robustly expressed within cardiac NCC populations and Dlx5/Dlx6 loss-of-function or gain-of-function mutants display no observable cardiac phenotypes [12,23-26]. Moreover, cardiac NCC expression of Dlx5 was observed in chicken [12,23-26] where differences between mammals were observed [35]. When DLX5 is expressed with cardiac NCC, although significant OFT abnormalities like PTA and DORV are observed, the expression of the Hand1 ${ }^{P A / O F T}$ enhancer is not clearly downregulated. This suggests that, although DLX5 is a dominate repressor in cranial NCC populations, its presence in cardiac NCCs does not impact Hand1 ${ }^{\text {PA/OFT }}$ enhancer activity, which suggests that DLX5 may not be sufficient for Hand1 transcriptional repression and that additional factors are required. Along these lines, it is important to note that HAND2 is necessary for Hand1 expression within cranial but not cardiac NCC. This also suggests that HAND2 must act with additional factors to regulate Hand1 within the cardiac NCCs. GATA transcription factors are also required for Hand1 ${ }^{\text {PA/OFT }}$ transcriptional activity [8]. In E10.5 reporter embryos in which GATA cis-regulatory elements in the Hand1 ${ }^{\text {PA/OFT }}$ enhancer have been mutagenized, cranial Hand $1^{\text {PA/OFT }}$ expression is ablated whereas cardiac NCC expression, although slightly reduced, persists [8]. Thus, factors required for enhancer activation in one NCC population (DLX5, SMAD, HAND2, GATA) do not significantly alter expression within another NCC population. Cranial NCC subpopulations share prepatterned chromatin states that are poised to respond to distinct local signaling cues depending upon where in the head they ultimately reside [40]. We propose that distinctions between cranial and cardiac NCC chromatin states enable these populations to respond to identical transcriptional inputs within the same cis-regulatory element in unique manners.

Given that there are observed OFT phenotypes, it is clear that ectopic DLX5 activity alters cardiac NCC gene expression. There is clear reduction in Hand2 expression within cardiac NCCs at E10.5 as well as a reduction of Sox 9 expressing cells (Figure 6). Changes in the cardiac NCC gene regulatory network combined with reductions in NCC numbers are the likely causes of the PTA and DORV observed in Dlx 5 NCC oe embryos. To assess potential NCC trans-differentiation, we looked at the neuronal markers TUBB3 and Ret, and found that, even though Dlx 5 expression is highly upregulated in Twist1-null cardiac NCCs, upregulation of DLX5 alone is insufficient to cause trans-differentiation (Figure 7).

Lastly, it is clear that $D l x$ gene dosage is modulated in Dlx 5 NCC oe mice. The highly related $D l x 6$, which is co-expressed with $D l x 5$ in cranial NCC, is significantly downregulated within the first and second pharyngeal arches of Dlx5 NCC oe embryos (Figure 8). A precise balance of specific transcription factors within subpopulations of NCCs appears necessary for these cells to migrate and differentiate to the correct tissue type and structures. NCC specification is thought to be governed from the rostral-caudal origin of delaminating NCCs from the neural tube. However, post migratory trans-differentiation is possible [13], which reflects the necessity for both positional and gene expression modulation. The data from this study reflects that altering the gene regulatory networks by transcription 
factor gain-of-function analysis can be used to reveal sensitive and insensitive actions of a single factor on a single enhancer in two separately fated populations of NCCs.

Author Contributions: Conceptualization, J.W.V. and A.B.F. Methodology, J.W.V., D.E.C., and A.B.F. Data curation, J.W.V. Original draft preparation, A.B.F. Writing, review editing, J.W.V., D.E.C., and A.B.F. Supervision, A.B.F. Funding, J.W.V., D.E.C., and A.B.F. All authors have read and agreed to the published version of the manuscript.

Funding: Infrastructural support at the Herman B. Wells Center for Pediatric Research is in part supported by the generosity of the Riley Children's Foundation, Division of Pediatric Cardiology, and the Carrolton Buehl McCulloch Chair of Pediatrics. This work is supported by the NIH P01HL134599 (ABF), 1R56DE029091 (ABF, DEC), and 1 R01HL145060 (ABF) and AHA 16SDG27260072 (JWV).

Acknowledgments: We thank Danny Carney, Beth Firulli, Kevin Toolan, and Tiffany Mundhenke for technical and experimental assistance. We thank the Herman B. Wells Center Cardiac Developmental Biology Group for helpful discussions. Infrastructural support at the Herman B. Wells Center is partially supported by the Riley Children's Foundation and the Carleton Buehl McCulloch Chair of Pediatrics.

Conflicts of Interest: The authors declare no conflicts of interest.

\section{References}

1. Hoffman, J.I.E. Incidence of congenital heart disease: II. Prenatal incidence. Pediatr. Cardiol. 1995, 16, 155-165. [CrossRef]

2. Schilling, T.F.; Le pabic, P. Neural Crest Cells; Academic Press: London, UK, 2014. [CrossRef]

3. Ruest, L.-B.; Dager, M.; Yanagisawa, H.; Charité, J.; Hammer, R.E.; Olson, E.N.; Yanagisawa, M.; Clouthier, D.E. dHAND-Cre transgenic mice reveal specific potential functions of dHAND during craniofacial development. Dev. Boil. 2003, 257, 263-277. [CrossRef]

4. Barbosa, A.C.; Funato, N.; Chapman, S.; McKee, M.D.; Richardson, J.A.; Olson, E.N.; Yanagisawa, H. Hand transcription factors cooperatively regulate development of the distal midline mesenchyme. Dev. Boil. 2007, 310, 154-168. [CrossRef]

5. Hendershot, T.J.; Liu, H.; Clouthier, D.E.; Shepherd, I.T.; Coppola, E.; Studer, M.; Firulli, A.B.; Pittman, U.L.; Howard, M.J. Conditional deletion of Hand2 reveals critical functions in neurogenesis and cell type-specific gene expression for development of neural crest-derived noradrenergic sympathetic ganglion neurons. Dev. Boil. 2008, 319, 179-191. [CrossRef] [PubMed]

6. Barron, F.; Woods, C.; Kuhn, K.; Bishop, J.; Howard, M.J.; Clouthier, D.E. Downregulation of Dlx5 and Dlx6 expression by Hand 2 is essential for initiation of tongue morphogenesis. Development 2011, 138, 2249-2259. [CrossRef] [PubMed]

7. George, R.M.; Firulli, A.B. Hand Factors in Cardiac Development. Anat. Rec. Adv. Integr. Anat. Evol. Boil. 2018, 302, 101-107. [CrossRef] [PubMed]

8. Vincentz, J.W.; Casasnovas, J.J.; Barnes, R.M.; Que, J.; Clouthier, D.E.; Wang, J.; Firulli, A.B. Exclusion of Dlx5/6 expression from the distal-most mandibular arches enables BMP-mediated specification of the distal cap. Proc. Natl. Acad. Sci. USA 2016, 113, 7563-7568. [CrossRef] [PubMed]

9. Firulli, B.A.; Fuchs, R.K.; Vincentz, J.W.; Clouthier, D.E.; Firulli, A.B. Hand1 phosphoregulation within the distal arch neural crest is essential for craniofacial morphogenesis. Development 2014, 141, 3050-3061. [CrossRef]

10. Bonilla-Claudio, M.; Wang, J.; Bai, Y.; Klysik, E.; Selever, J.; Martin, J.F. Bmp signaling regulates a dose-dependent transcriptional program to control facial skeletal development. Development 2012, 139, 709-719. [CrossRef]

11. Liu, W.; Selever, J.; Murali, D.; Sun, X.; Brugger, S.M.; Ma, L.; Schwartz, R.J.; Maxson, R.; Furuta, Y.; Martin, J.F. Threshold-specific requirements for Bmp4 in mandibular development. Dev. Boil. 2005, 283, 282-293. [CrossRef]

12. Soldatov, R.; Kaucká, M.; Kastriti, M.E.; Petersen, J.; Chontorotzea, T.; Englmaier, L.; Akkuratova, N.; Yang, Y.; Häring, M.; Dyachuk, V.; et al. Spatiotemporal structure of cell fate decisions in murine neural crest. Science 2019, 364, eaas9536. [CrossRef] [PubMed]

13. Vincentz, J.W.; Firulli, B.A.; Lin, A.; Spicer, U.B.; Howard, M.J.; Firulli, A.B. Twist1 Controls a Cell-Specification Switch Governing Cell Fate Decisions within the Cardiac Neural Crest. PLoS Genet. 2013, 9, e1003405. [CrossRef] [PubMed] 
14. Vincentz, J.W.; VanDusen, N.J.; Fleming, A.B.; Rubart, M.; Firulli, B.A.; Howard, M.J.; Firulli, A.B. A Phox2and Hand2-dependent Hand1 cis-regulatory element reveals a unique gene dosage requirement for Hand2 during sympathetic neurogenesis. J. Neurosci. 2012, 32, 2110-2120. [CrossRef] [PubMed]

15. Connerney, J.; Andreeva, V.; Leshem, Y.; Muentener, C.; Mercado, M.A.; Spicer, D.B. Twist1 dimer selection regulates cranial suture patterning and fusion. Dev. Dyn. 2006, 235, 1334-1346. [CrossRef]

16. Barnes, R.M.; Firulli, B.A.; Conway, S.J.; Vincentz, J.W.; Firulli, A.B. Analysis of the Hand1 cell lineage reveals novel contributions to cardiovascular, neural crest, extra-embryonic, and lateral mesoderm derivatives. Dev. Dyn. 2010, 239, 3086-3097. [CrossRef]

17. VanDusen, N.J.; Casanovas, J.; Vincentz, J.W.; Firulli, B.A.; Osterwalder, M.; Lopez-Rios, J.; Zeller, R.; Zhou, B.; Grego-Bessa, J.; De La Pompa, J.L.; et al. Hand2 is an essential regulator for two Notch-dependent functions within the embryonic endocardium. Cell Rep. 2014, 9, 2071-2083. [CrossRef]

18. Firulli, B.A.; Milliar, H.; Toolan, K.P.; Harkin, J.; Fuchs, R.K.; Robling, A.G.; Firulli, A.B. Defective Hand1 phosphoregulation uncovers essential roles for Hand1 in limb morphogenesis. Development 2017, 144, 2480-2489. [CrossRef]

19. Vincentz, J.; Toolan, K.P.; Zhang, W.; Firulli, A.B. Hand factor ablation causes defective left ventricular chamber development and compromised adult cardiac function. PLoS Genet. 2017, 13, e1006922. [CrossRef]

20. Vincentz, J.W.; Barnes, R.M.; Rodgers, R.; Firulli, B.A.; Conway, S.J.; Firulli, A.B. An absence of Twist1 results in aberrant cardiac neural crest morphogenesis. Dev. Boil. 2008, 320, 131-139. [CrossRef]

21. Barnes, R.M.; Firulli, B.A.; VanDusen, N.J.; Morikawa, Y.; Conway, S.J.; Cserjesi, P.; Vincentz, J.W.; Firulli, A.B. Hand2 loss-of-function in Hand1-expressing cells reveals distinct roles in epicardial and coronary vessel development. Circ. Res. 2011, 108, 940-949. [CrossRef]

22. Danielian, P.; Muccino, D.; Rowitch, D.H.; Michael, S.K.; McMahon, A.P. Modification of gene activity in mouse embryos in utero by a tamoxifen-inducible form of Cre recombinase. Curr. Boil. 1998, 8, 1323-S2. [CrossRef]

23. Shimizu, M.; Narboux-Nême, N.; Gitton, Y.; De Lombares, C.; Fontaine, A.; Alfama, G.; Kitazawa, T.; Kawamura, Y.; Heude, E.; Marshall, L.; et al. Probing the origin of matching functional jaws: Roles of Dlx5/6 in cranial neural crest cells. Sci. Rep. 2018, 8, 14975. [CrossRef] [PubMed]

24. Robledo, R.F.; Rajan, L.; Li, X.; Lufkin, T. The Dlx5 and Dlx6 homeobox genes are essential for craniofacial, axial, and appendicular skeletal development. Genome Res. 2002, 16, 1089-1101. [CrossRef] [PubMed]

25. Tavares, A.; Clouthier, D.E. Cre recombinase-regulated Endothelin1 transgenic mouse lines: Novel tools for analysis of embryonic and adult disorders. Dev. Boil. 2015, 400, 191-201. [CrossRef]

26. Tani-Matsuhana, S.; Vieceli, F.; Gandhi, S.; Inoue, K.; E Bronner, M. Transcriptome profiling of the cardiac neural crest reveals a critical role for MafB. Dev. Boil. 2018, 444, S209-S218. [CrossRef]

27. Minoux, M.; Rijli, F.M. Molecular mechanisms of cranial neural crest cell migration and patterning in craniofacial development. Development 2010, 137, 2605-2621. [CrossRef]

28. Holler, K.L.; Hendershot, T.J.; Troy, S.E.; Vincentz, J.W.; Firulli, A.B.; Howard, M.J. Targeted deletion of Hand2 in cardiac neural crest-derived cells influences cardiac gene expression and outflow tract development. Dev. Boil. 2010, 341, 291-304. [CrossRef]

29. Tsuchihashi, T.; Maeda, J.; Shin, C.H.; Ivey, K.N.; Black, B.L.; Olson, E.N.; Yamagishi, H.; Srivastava, D. Hand2 function in second heart field progenitors is essential for cardiogenesis. Dev. Boil. 2010, 351, 62-69. [CrossRef]

30. Cheung, M.; Briscoe, J. Neural crest development is regulated by the transcription factor Sox9. Development 2003, 130, 5681-5693. [CrossRef]

31. Sahar, D.E.; Longaker, M.T.; Quarto, N. Sox 9 neural crest determinant gene controls patterning and closure of the posterior frontal cranial suture. Dev. Boil. 2005, 280, 344-361. [CrossRef]

32. Sullivan, K.; Cleveland, D.W. Identification of conserved isotype-defining variable region sequences for four vertebrate beta tubulin polypeptide classes. Proc. Natl. Acad. Sci. USA 1986, 83, 4327-4331. [CrossRef]

33. Beck, K.D.; Valverde, J.; Alexi, T.; Poulsen, K.; Moffat, B.; Vandlen, R.A.; Rosenthal, A.; Hefti, F. Mesencephalic dopaminergic neurons protected by GDNF from axotomy-induced degeneration in the adult brain. Nature 1995, 373, 339-341. [CrossRef] [PubMed]

34. Ruest, L.-B.; Xiang, X.; Lim, K.-C.; Levi, G.; Clouthier, D.E. Endothelin-A receptor-dependent and -independent signaling pathways in establishing mandibular identity. Development 2004, 131, 4413-4423. [CrossRef] 
35. Vincentz, J.W.; Firulli, A.B. The Cardiac Neural Crest and Their Role in Development and Disease; Elsevier BV: London, UK, 2014; Volume 1, pp. 205-229.

36. Firulli, A.B.; McFadden, D.G.; Lin, Q.; Srivastava, D.; Olson, E.N. Heart and extra-embryonic mesodermal defects in mouse embryos lacking the bHLH transcription factor Hand1. Nat. Genet. 1998, 18, 266-270. [CrossRef] [PubMed]

37. Srivastava, D.; Thomas, T.; Lin, Q.; Kirby, M.L.; Brown, R.; Olson, E.N. Regulation of cardiac mesodermal and neural crest development by the bHLH transcription factor, dHAND. Nat. Genet. 1997, 16, 154-160. [CrossRef] [PubMed]

38. McFadden, D.G.; Barbosa, A.C.; Richardson, J.A.; Schneider, M.D.; Srivastava, D.; Olson, E.N. The Hand1 and Hand2 transcription factors regulate expansion of the embryonic cardiac ventricles in a gene dosage-dependent manner. Development 2004, 132, 189-201. [CrossRef] [PubMed]

39. Charité, J.; McFadden, D.G.; Merlo, G.; Levi, G.; Clouthier, D.E.; Yanagisawa, M.; Richardson, J.A.; Olson, E.N. Role of Dlx6 in regulation of an endothelin-1-dependent, dHAND branchial arch enhancer. Genome Res. 2001, 15, 3039-3049. [CrossRef]

40. Minoux, M.; Holwerda, S.; Vitobello, A.; Kitazawa, T.; Kohler, H.; Stadler, M.B.; Rijli, F.M. Gene bivalency at Polycomb domains regulates cranial neural crest positional identity. Science 2017. [CrossRef]

(C) 2020 by the authors. Licensee MDPI, Basel, Switzerland. This article is an open access article distributed under the terms and conditions of the Creative Commons Attribution (CC BY) license (http://creativecommons.org/licenses/by/4.0/). 